\title{
Lepadarg
}

- sur

URBANISME RÉGULIER, OCCUPATION DES TERRES ET RAPPORTS INTERETHNIQUES

EN GRANDE-GRÈCE

REGULAR URBANISM, LAND OCCUPATION AND INTERETHNICAL RELATIONS IN

MAGNA GRAECIA

(Urbanismo regular, ocupação das terras e relações interétnicas na Magna Grécia)

Airton Pollini 


\title{
Urbanisme régulier, occupation des terres et rapports interethniques en Grande- Grèce
}

\begin{abstract}
Airton Pollini ${ }^{1}$
Résumé : L'Italie du Sud est probablement la région la mieux connue du monde grec colonial antique. Quelques sources écrites mais surtout des études archéologiques menées depuis longtemps ont permis le développement des recherches sur plusieurs aspects au cœur de la thématique de la colonisation grecque. Ce travail se concentre sur trois aspects essentiels : l'appropriation de l'espace colonial, l'interaction avec les populations indigènes et l'urbanisation des nouvelles installations.
\end{abstract}

Mots-clés : Grande-Grèce ; Espace ; Indigènes ; Urbanisation.

Resumo: O Sul da Itália é provavelmente a região mais bem conhecida do Mundo Grego Antigo. Algumas fontes escritas, mas principalmente os estudos arqueológicos, realizados desde um longo tempo permitiram o desenvolvimento de pesquisas sobre vários aspectos no cerne da temática da colonização grega. Este trabalho se concentra em três aspectos essenciais: a apropriação do espaço colonial, a interação com as populações indígenas e a urbanização das novas instalações.

Palavras-chave: Magna Grécia; Espaço; Indígenas; Urbanização.

\begin{abstract}
The South Italy is probably the best known region of the ancient Greek colonial world. Some written sources but especially archaeological work undertaken for a long time allowed the development of research on several aspects at the heart of the issues of Greek colonization. This paper concentrates on three essential aspects: the appropriation of colonial space, the interaction with the Natives, and the urbanization of new establishments.
\end{abstract}

Keywords: Magna Graecia; Space Natives; Urbanization.

\section{LA GRANDE-GRECE}

La région qui s'étend de la baie de Naples jusqu'aux bouts de la péninsule italienne est connue par les spécialistes d'histoire grecque par la dénomination « Grande-Grèce ». Cette expression est la traduction littérale des termes grecs Megale Hellas, dont la version en latin est Magna Graecia ou bien Graecia Maior (cf. Atti Taranto 1981 MUSTI, 1988). Pourtant, l'origine grecque de l'expression ne résout pas la difficulté de sa définition. L'extension de l'espace couvert par la Megale Hellas n'était pas toujours la même selon la source antique considérée. En effet, on a pu montrer que la Grande-Grèce était un concept créé, après la victoire de la cité de Crotone sur sa voisine Sybaris en 511 av. J.-C., par la tradition d'origine crotoniate, très probablement développée dans les cercles pythagoriciens influents dans cette cité (cf. AMERUOSO, 1996).

\footnotetext{
${ }^{1}$ Maître de conférences d'histoire de l'Antiquité grecque à l'Université de Haute-Alsace (Université de Haute-Alsace, Université de Strasbourg, CNRS ArcHiMedE UMR 7044 Mulhouse), France.
} 
Ainsi, au V siècle av. J.-C., la Grande-Grèce devait correspondre à l'espace délimité par un triangle formé par les cités de Métaponte vers l'est, Poseidonia à l'ouest et Rhégion (actuelle Reggio di Calabria) à l'extrémité sud de la péninsule. Le fait même que les points de délimitation forment un triangle est un élément à la base de l'identification de son origine probablement pythagoricienne. Ainsi, les cités grecques de la baie de Naples (Pithécusses, Cumes et Naples - voir l'article d'A. Esposito dans ce dossier), ainsi que Tarente restaient en dehors. Aujourd'hui, en revanche, les spécialistes utilisent l'expression pour l'ensemble des cités coloniales grecques de l'Italie du Sud, à l'exception de la Sicile, indépendamment de l'extension donnée par les sources grecques antiques.

De plus, les sources les plus anciennes montrent que la première appellation pour les colonies grecques du sud de l'Italie était «Italia » (LOMBARDO, 2001 et 2011 plus généralement sur la géographie antique de l'Italie méridionale, en particulier sur Strabon, voir GRECO, 1987 ; MADDOLI, 1987 ; PRONTERA, 1987 ; BIFFI, 1988 ; JANNI, 1988), même si, au départ, cette dénomination ne recouvrait qu'une partie de la Calabre actuelle, probablement en lien avec l'idée de l'« empire » de Sybaris (voir ci-dessous). C'est de façon progressive que l'Italia s'est élargie pour englober finalement l'ensemble de la péninsule et ce dès la période romaine. Cette première appellation explique pourquoi les Anciens se référaient aux Grecs de ces colonies comme étant des Italiotes, terme que l'on continue d'utiliser dans les livres d'histoire.

\section{L'URBANISME REGULIER EN OCCIDENT}

La définition même d'une cité-État, la polis grecque, comprend deux aspects, soit une communauté de citoyens, soit son expression physique dans une agglomération urbaine entourée des terres cultivées (voir des discussions approfondies dans HANSEN, 2001 ; HANSEN et NIELSEN, 2004 ; aussi nos commentaires dans POLLINI, 2015). Nous allons nous concentrer tout d'abord sur la question de la ville, et plus particulièrement sur les premiers cas connus d'une régularisation de l'occupation urbaine par les Grecs d'Occident (sur I'urbanisme grec en général, voir MARTIN, 1974 et GRECO et TORELLI, 1983, ainsi qu'une synthèse bibliographique plus récente GRECO, 2004-2005 et les synthèses LAFON, MARC et SARTRE, 2011 ; HELLMANN, 2010 ; voir aussi nos commentaires sur les représentations de la ville grecque d'Occident dans POLLINI, 2017).

Même si l'objectif de cette contribution est de se concentrer sur les exemples de Grande-Grèce, il est essentiel de faire une mention des cas siciliens qui constituent en grande mesure le point névralgique des débats. Ainsi, la situation à Syracuse est très suggestive (voir l'article de B. Florenzano).

En effet, la ville de Syracuse montre des traces d'axes urbains archaïques avec une relative régularité (TRÉZINY, 2002, sur Syracuse, p. 273-278). Le premier habitat grec de la colonie semble avoir été créé par le regroupement de villages (DI VITA, 1996, p. 270-274), aussi bien sur l'île d'Ortygie que sur la partie 
proprement sicilienne à Achradine, où ont été trouvés les vestiges les plus anciens de présence grecque dans la nécropole de la zone de Fusco. Assurément, l'établissement des Corinthiens a intégré un certain nombre de centres indigènes avec leur population et l'axe principal nord-sud qui traverse Ortygie a fonctionné tout d'abord pour relier les divers centres d'occupation de la petite île et, par extension, de la partie sicilienne d'Achradine. Cet axe central a été ensuite complété par une série de voies transversales, dans une organisation raisonnée, assez régulière mais qui n'est pas orthogonale (HELLMANN, 2010, p. 188-189 ; MERTENS, 2006, p. 73-76). En effet, l'angle formé par l'intersection des axes principaux et les voies transversales n'est pas droit, mais d'environ 79 degrés (Fig. 2).

Dans l'état actuel de nos connaissances, ce système urbain peut être daté avec certitude entre la fin du VII et le début du VI siècle av. J.-C. sans que l'on puisse établir la forme de l'espace urbain au moment de la fondation de la colonie de Syracuse dans le dernier tiers du VIII siècle av. J.-C. L'hypothèse largement retenue est d'un établissement fonctionnel des espaces de Syracuse dès sa fondation (LA TORRE, 2011, p. 171-173).

Si le cas de Syracuse montre une volonté certaine de régularisation de l'occupation de l'espace urbain dès la fin du VII siècle, l'exemple paradigmatique de l'urbanisation régulière grecque est celui de Mégara Hyblaea (DE ANGELIS, 2003, p. 17-39 ; GRAS, TRÉZINY et BROISE, 2004, p. 523-526 ; MERTENS, 2006, p. 63-72). Les recherches anciennes et récentes ont montré un schéma d'organisation de l'espace urbain très régulier, bien que non orthogonal. Deux voies principales ( $A$ et $B$, mesurant entre 5,30 à 5,80 m de largeur) structurent l'espace dans le sens est-ouest. Ces « avenues » parcourent l'ensemble de la ville et relient d'une part l'une des portes des remparts au bord de mer (avenue B), d'autre part le sanctuaire occidental à l'agora (avenue A). Bien qu'organisant de façon ordonnée l'espace, ces deux voies ne sont pas parfaitement parallèles et les différences dans l'écart entre elles sont probablement dues à une volonté de division équilibrée de l'espace compris entre l'avenue B et le rempart nord (GRAS, TRÉZINY et BROISE, 2004, p. 527530). Puis, un quadrillage composé de trois groupes de voies de circulation, parallèles entre elles ( $C, D$ et $E$ ), en direction nord-sud et d'une largeur d'environ $3 \mathrm{~m}$, aménage l'ensemble de la ville. Les différents groupes de voies portent des écarts d'orientation, donnant lieu à un système « oblique » (SHIPLEY, 2005, p. 341), ou bien d'un «plan régulier par secteur » (TRÉZINY, 2002, p. 267-272), laissant, dans la partie centrale du plateau, une zone non divisée de forme trapézoïdale identifiée comme l'agora (GRAS, TRÉZINY et BROISE, 2004, p. 391-445 ; MERTENS, 2006, p. 67-69). Les îlots d'habitations ont des superficies assez régulières d'environ $25 \mathrm{~m}$ de large pour $98 \mathrm{~m}$ à $110 \mathrm{~m}$ de long.

La chronologie actuellement acceptée à partir des données archéologiques disponibles place ce système urbain comme le plus ancien cas connu, établi dès les environs de $700 \mathrm{av}$. J.-C., c'est-à-dire à moins d'une génération de la fondation de la cité, mais avec une matérialisation plus importante dans une seconde 
phase, vers 640-630 av. J.-C. (GRAS, TRÉZINY et BROISE, 2004, p. 237-301, en particulier les conclusions p. 301).

L'explication d'H. Tréziny pour le schéma d'urbanisme de Mégara est fondée sur un module de lot urbain individuel standard (oikopedon), comprenant entre 120 et $140 \mathrm{~m}^{2}$ de superficie (TRÉZINY, 1999, p. 141-183 ; GRAS, TRÉZINY et BROISE, 2004, p. 532-539). Si tous les lots urbains n'ont pas été occupés dès le départ, la structure urbaine a été définie dès les premiers temps de la colonie. S'il y a des variations relativement réduites dans la superficie des lots urbains, mesurées par les recherches archéologiques, elles n'invalident pas l'idée d'un système de distribution de lots selon un principe d'égalité. En tout cas, l'urbanisme de Mégara Hyblaea est fondé sur les principes de régularisation de l'espace et d'égalité du lotissement, avec des îlots de superficie presque identiques.

Enfin, le dernier exemple sicéliote paradigmatique est celui de Sélinonte, la sous-colonie de Mégara Hyblaea, fondée dans I'Ouest de la Sicile au VII siècle, soit vers 651 av. J.-C. d'après Diodore de Sicile (XIII, 59), soit plus probablement vers 628 av. J.-C. d'après Thucydide (VI, 4) (sur l'urbanisme de Sélinonte, voir en particulier les synthèses MERTENS, 2006, p. 83-85 et DE ANGELIS, 2003, p. 128-145). En opposition à I'interprétation de G. Vallet (VALLET, 1983, p. 646), M. Gras, H. Tréziny et H. Broise rapprochent les deux colonies dans leur organisation de l'espace urbain, en affirmant que la principale différence entre les deux villes est l'emploi de l'angle droit à Sélinonte (GRAS, TRÉZINY et BROISE, 2004, p. 589). L'autre différence essentielle est leur taille, puisque d'une superficie totale d'environ 60 hectares pour Mégara, on passe à environ 130 à Sélinonte et les dimensions des lots standards (oikopeda) sont presque doubles, d'environ $120 \mathrm{~m}^{2}$ dans le premier cas, ils passent à environ $210 \mathrm{~m}^{2}$ dans le second. Mais la chronologie est l'élément essentiel. En effet, l'urbanisme régulier de Sélinonte est daté dans les années 580-570 av. J.-C., ce qui le place à la condition du plus ancien cas connu d'un urbanisme grec orthogonal (cf. SHIPLEY, 2005, p. 342).

Une fois établis les principes d'un urbanisme orthogonal dès le début du VI siècle av. J.-C., plusieurs exemples concrets sont connus en Occident, en particulier en Grande-Grèce, comme notamment à Métaponte ou à Poseidonia (DE SIENA, 1991 MERTENS, 1991 GRECO, 2000). Le cas plus récent de Neapolis est désormais assez bien connu (cf. en dernier LONGO et TAURO, 2017).

En revanche, un exemple qui mérite notre attention est celui de Cumes. Les travaux de M. D'Acunto, entrepris depuis 2007 (D'ACUNTO, 2009; D'ACUNTO, 2014. Voir aussi D'AGOSTINO et D'ACUNTO, 2008), ont dégagé un quartier résidentiel situé entre la zone du forum romain et les remparts de la ville. En effet, l'urbanisme de Cumes montre une organisation dite "raisonnée ", à défaut d'être orthogonale (cf. D'ONOFRIO, 2002) : l'espace urbain s'organise à partir d'une grande plateia (" avenue ») nord-sud qui part de la zone du forum, probablement à l'emplacement de l'ancienne agora grecque, en direction des remparts septentrionaux et de la dite « Porta mediana », avec des stenopoi (" rues ») réguliers. 
Ce système n'est pas orthogonal par rapport à la plateia, car l'avenue elle-même n'est pas rectiligne, puisqu'elle présente une sorte de crochet.

L'élément le plus intéressant est la chronologie, très haute, dès la fin du VIII et dans le courant du VII siècle. De ces phases plus anciennes, ont été trouvées surtout des strates, dont peut-être un niveau de circulation, quelques traces d'un habitat, et du matériel céramique (D'ACUNTO, 2014, p. 23). Dès le VIe siècle, les vestiges sont plus importants, dont deux habitations, qui attestent, à travers la découverte de la vaisselle de symposion de très haute qualité, la présence d'un groupe socialement très élevé ici. II est essentiel de remarquer que l'exemple de Cumes permet d'affirmer qu'un urbanisme raisonné et régulier peut, tout de même, donner lieu à des différenciations sociales importantes, avec l'installation des couches aisées dans un espace divisé de façon probablement égalitaire au départ (cf. POLLINI, à paraître). À titre de comparaison, il est intéressant de mettre les cas de Cumes et de Milet en parallèle. En effet, à Milet, le plan régulier date de la fin du $\mathrm{Vl}^{\mathrm{e}}$ siècle, œuvre des tyrans. La reconstruction de la ville après les victoires contre les Perses et sous un régime démocratique n'a fait que suivre les axes déterminés précédemment, ce qui écarte tout lien entre un urbanisme régulier et des principes d'égalité démocratique (ASHERI, 1975 ; WEBER, 2007 ; HELLMANN, 2010).

Tout commentaire sur l'idée d'urbanisme régulier en Grande-Grèce doit évoquer le cas emblématique de Thourioi, cité panhellénique fondée sur l'emplacement de l'ancienne Sybaris, sous I'impulsion de l'Athènes de Périclès, vers 444 av. J.-C. (cf. GRECO, 2009 ; GRECO et LUPPINO, 1999 ; GRECO, LUPPINO, et al., 2010 ; MARINO, 2010)². À propos de Thourioi, notre principale source écrite est Diodore de Sicile (Bibliothèque historique, XII, 10, 6-7. Cf. GRECO, 1999, p. 415 ; GRECO, 1999 ; GRECO, 1999 ; GARCÍA QUINTELA, 2000). L'historien de Sicile s'intéresse surtout à la législation de la nouvelle cité, mais décrit également l'organisation spatiale de la fondation coloniale. La ville suit ainsi un schéma régulier avec quatre voies principales (plateiai) dans un sens et trois dans l'autre, puis une série de voies parallèles (stenopoi). Le plan était parfaitement orthogonal et, de surcroît, le chiffre sept coïncide avec le nombre de cordes d'une lyre, probablement en référence à l'idéal de perfection lié aux courants pythagoriciens très actifs dans la région.

Le cas de Thourioi est particulièrement intéressant pour la mise en parallèle du récit écrit et des données archéologiques observées sur le terrain (CASTAGNOLI, 1971; CASTAGNOLI, 1973; pour les recherches récentes sur l'urbanisme de Thourioi, voir GRECO et LUPPINO, 1999). Actuellement, il est possible

\footnotetext{
${ }^{2}$ On ne traitera pas ici des discussions sur la participation d'Hippodamos de Milet dans l'établissement du plan d'urbanisme de Thourioi. L'ensemble des sources sur Hippodamos est recueilli dans SHIPLEY, 2005, p. 356-361 et 386388. À propos d'Hippodamos, voir notamment HELLMANN, 2010, p. 191-197; CALIÒ, 2012, p. 105-126. Sur l'agora " hippodaméenne » du Pirée, voir LONGO, 2008, p. 137-155 et LONGO, 2014, p. 217-231. Sur la participation d'autres intellectuels célèbres dans la fondation de Thourioi (Diogène Laërce, IX, 50 = Protagoras), voir BERTELLI, 1997, p. 108121 et GARCÍA QUINTELA, 2006, p. 199-222 sur la participation d'Hérodote, voir nos commentaires dans POLLINI, 2009.
} 
de restituer cinq des sept avenues mentionnées par Diodore et, par extrapolation, en reportant des écarts réguliers entre les voies principales, E. Greco a pu reconstituer (Fig. 3), à titre d'hypothèse pour le moment, l'ensemble du plan de Thourioi (GRECO, 2009 et GRECO, LUPPINO, et al., 2010). II serait composé d'un ensemble rectangulaire de 396 m par 296 m (1300 x 1000 pieds), divisé en carrés de 37 m de côté qui pourraient accueillir, chacun, quatre maisons de $18 \mathrm{~m}$ par $18 \mathrm{~m}\left(324 \mathrm{~m}^{2}\right)$, des mesures comparables à d'autres exemples contemporains, comme Olynthe en Grèce septentrionale (CAHILL, 2002) et Himère en Sicile (VASSALLO, 2005 ; ALLEGRO, 2008). Il s'agit ici du cas le plus abouti d'une organisation régulière et parfaitement orthogonale de l'espace, attesté autant par les sources écrites que par les vestiges archéologiques.

Les recherches récentes au-delà de la porte septentrionale portent à croire à une prolongation des grands axes également dans la zone rurale, ce qui mène à l'hypothèse selon laquelle la régularité de l'organisation de l'espace à Thourioi ne se limitait pas à la partie urbaine.

\section{L'APPROPRIATION DES TERRES EN GRANDE-GRECE}

Les recherches archéologiques sur la zone rurale sont aujourd'hui un aspect essentiel et reconnu par tous. En revanche, c'est d'abord dans le domaine colonial, en mer Noire à Chersonèse de Crimée, en Sicile à Géla (voir l'article de B. Florenzano sur Syracuse), puis en Italie du Sud, d'abord à Métaponte et à Poseidonia, que les recherches se sont développées, respectivement, dès les années 1940, 1950 et 1960 (cf. OSANNA, 1992 ; POLLINI, 2006 ; POLLINI, 2012). À titre de comparaison, les études systématiques du milieu rural en Grèce égéenne n’ont commencé que dans les années 1980 (BINTLIFF, 1994 ; BINTLIFF, 1999 ; BRUNET, 2000). C'est dans le cadre de ces recherches, réalisées dans les zones plus ou moins éloignées des centres urbains antiques, que l'appropriation de l'espace par l'arrivée des colons peut être perçue. Et un signe très représentatif peut être reconnu dans l'établissement de sanctuaires extra urbains dans des lieux symboliques pour le contrôle de l'espace par la communauté des colons (DE POLIGNAC, 1995 ; discussion récente dans DAVIES, 2013, p. 18-20).

Tout commentaire sur les cités grecques de Grande-Grèce, en excluant la région de la baie de Naples, doit commencer par la cité de Sybaris (Atti Taranto 1992 ; DELIA et MASNERI, 2013), une des premières fondations, dès les années 720 av. J.-C. Le géographe Strabon (Géographie, VI, 1, 13) écrit que la cité était si puissante qu'elle pouvait commander à quatre " peuples » (ethne) voisins, avoir vingt-cinq cités (poleis) sujettes et avoir des remparts circulaires de 50 stades de longueur $(9,25 \mathrm{~km})$. Outre sa richesse proverbiale, ce témoignage montre que la cité avait une claire volonté de constituer une sorte de zone d'influence bien au-delà des limites du territoire appartenant aux citoyens et exploité par eux (Fig. 4). Les quatre peuples et les vingt-cinq communautés voisines ne peuvent évidemment pas être identifiés 
précisément, mais il s'agit certainement de centres de populations indigènes ayant des rapports très étroits avec la cité grecque de Sybaris (BUGNO, 2001 fait le parallèle avec le modèle oriental d'hégémonie par districts établi par les Perses). L'analyse des vestiges archéologiques des sites de Francavilla Marittima (MAASKANT-KLEIBRINK, 2005 ; GRANESE, 2006 ; QUONDAM, 2009 ; LUPPINO, QUONDAM, et al., 2010 ; GRANESE, 2013 ; GUZZO, 2013 ; BROCATO, 2014 ; GUGGISBERG, 2016 ; QUONDAM, 2016), où un sanctuaire de type grec est érigé à la place d'un établissement indigène préexistant, et d'Amendolara (LA GENIÈRE DE, 1984 ; LA GENIÈRE DE, 2012), qui reste un site plutôt indigène avec d'importants éléments grecs, montre deux situations bien différentes dans une même aire géographique et culturelle.

Les volontés expansionnistes de Sybaris au-delà de son territoire civique ont été interprétées comme l'un des premiers essais de formation d'un type d'« empire», dans le sens d'un large réseau d'alliances et d'influences qui devait être à l'origine de la notion d'Italia et qui a pu englober la majeure partie de la Grande-Grèce, c'est-à-dire de la région des cités grecques en Italie du Sud (GRECO, 1992 ; BUGNO, 2001 ; GRECO, 2013). De plus, on a pu établir une association de cette idée d'« empire de Sybaris » non seulement avec les alliances tournées vers les centres indigènes, mais aussi avec la fondation d'autres cités coloniales grecques, comme Métaponte (vers 630), Poseidonia (vers 600), voire Vélia (vers 540). Nous considérons que cette zone d'influence très élargie de Sybaris fait partie d'un essai de territorialisation, et donc d'un certain contrôle de l'espace colonial, dans le sens le plus large possible. Ainsi, peut-être la notion primitive de l'Italia grecque était un fait des volontés impérialistes de Sybaris dans le sens de l'identification d'une certaine unité des cités grecques de la région sous son hégémonie.

En tout cas, les volontés expansionnistes de Sybaris se sont heurtées à l'opposition de la cité voisine Crotone, fondée à peu près au même moment, vers la fin du VIII e siècle av. J.-C. Si les cités coloniales pouvaient former des réseaux d'alliances entre elles, le conflit qui a abouti à la destruction complète de Sybaris entre 511 et 510 av. J.-C. montre la variété des rapports entre les différents acteurs en domaine colonial, notamment les différents groupes de colons et les indigènes (cf. LOMBARDO, 2002). Après la chute de Sybaris, il semblerait qu'une grande partie des Sybarites survivants se soient transférés sur la côte tyrrhénienne pour fonder une autre colonie, celle de Laos à l'extrême fin du $\mathrm{Vl}^{\mathrm{e}}$ siècle.

Dans l'analyse de l'appropriation de l'espace rural, la cité de Métaponte mérite une place d'honneur : c'est le cas le mieux connu du territoire d'une cité dans l'ensemble du monde grec. Des traces visibles grâce aux photographies aériennes, vérifiées sur le terrain par des fouilles stratigraphiques systématiques dès les années 1960 (SCHMIEDT et CHEVALLIER, 1959 ; SCHMIEDT et CHEVALLIER, 1960 ; ADAMESTEANU, 1965), puis des prospections récentes menées par les équipes américaines de l'université du Texas sous la direction de J. C. Carter ont produit des données exceptionnelles (CARTER, 1998 ; CARTER, 2006 ; CARTER et PRIETO, 2011). II s'agit de traces d'une division régulière de l'espace rural (Fig. 5), matérialisée par un réseau de canalisations parallèles qui définissent des lots de terres de taille égale (Fig. 
6). Si un tel système est aussi connu en Chersonèse de Crimée en mer Noire pour le IV siècle av. J.-C., à Métaponte, les vestiges les plus anciens de cette organisation des terres datent d'environ 580, c'est-à-dire entre une et deux générations après l'arrivée des colons achéens. Les sources numismatiques corroborent l'importance de la campagne de Métaponte puisque la cité représentait un épi d'orge sur ses propres monnaies (STAZIO, 1973) (Fig. 7).

Ces deux exemples célèbres de Sybaris et de Métaponte permettent de souligner plusieurs aspects. D'une part, à l'arrivée des colons, l'espace n'était pas vide, comme l'on pourrait croire par l'utilisation de l'expression grecque eremos chora (littéralement " territoire désert »). Cette expression doit être prise à partir du point de vue grec: il s'agirait de terres dépourvues d'une forme grecque (ou comparable) d'organisation, la cité-État, mais aucunement d'un territoire désert. D'autre part, l'appropriation de l'espace par les colons peut se faire soit aux dépens des populations, soit avec une certaine forme de cohabitation cela peut être le résultat de l'établissement de petits villages disséminés dans la campagne (kata komas), comme c'est probablement le cas pour Sybaris, ou bien par une occupation capillaire des terres, comme à Métaponte.

\section{RAPPORTS INTERETHNIQUES ET LA PREMIÈRE FORME CONNUE DE « DÉCOLONISATION »}

Les cas déjà évoqués de l'« empire » de Sybaris, ainsi que l'analyse des phases dites précoloniales dans la région du golfe de Tarente (Siritide e Metapontino 1991 ; PERONI et TRUCCO, 1994 ; ESPOSITO, 2005 ; voir l'article d'A. Esposito dans ce dossier), montrent très clairement la variété des situations dans l'interaction entre colons grecs et populations indigènes.

Outre les colons grecs installés dans la partie la plus méridionale de la péninsule, les Étrusques se sont également installés dans les terres de l'actuelle Campanie, dans un mouvement comparable à celui des Grecs (CERCHIAI, 2008 et 2011 ; D'AGOSTINO, 2011). D'autre part, la Grande-Grèce montre un cas unique : la première forme connue de " décolonisation », pour reprendre l'expression devenue célèbre de $\mathrm{D}$. Asheri (ASHERI, 1996). À partir du milieu du V' siècle av. J.-C., des populations italiques, des Campaniens et des Lucaniens d'origine samnite (PONTRANDOLFO, 1982; CERCHIAI, 1995), ont poursuivi un mouvement d'expansion vers le sud de la péninsule. Ce mouvement a abouti à la conquête de certaines cités grecques par ces populations : les Campaniens s'emparent de Naples dès le milieu du Ve siècle (CERCHIAI, 2010), puis les Lucaniens se font maîtres des cités tyrrhéniennes de Poseidonia dès les années 420-410 et de Laos vers 390 av. J.-C. (GRECO, GRECO et PONTRANDOLFO, s.d. ; Atti Taranto 1987 ; PONTRANDOLFO et D'AGOSTINO, 1987 ; CIPRIANI et LONGO, 1996 ; ROUVERET, 2012). Ensuite, même sans réussir à prendre possession des autres cités grecques, ils occupent l'ensemble de l'arrière-pays de l'Italie du Sud (actuelles régions de Basilicate et de Calabre) et exercent une pression très forte sur les Grecs d'Italie. II s'agit ainsi du premier cas 
connu d'un renversement du rapport des forces, où l'élément local, italique, bien que venu d'une région plus au nord, est capable de mettre sous son hégémonie une partie des colons grecs. Il faut peut-être mettre cette réalité en parallèle avec les " cadeaux ", forme déguisée d'une sorte de tribut, que certaines cités grecques de mer Noire devaient aux populations indigènes qui les entouraient (voir l'article de M. Dana dans ce dossier). Si la pression militaire sur les cités grecques du Pont Euxin est évidente, le cas de la Grande-Grèce est le seul exemple connu où les populations locales soumettent complètement les Grecs et contrôlent directement la cité conquise.

L'affaiblissement relatif des cités grecques d'Italie est aussi perceptible par l'appel que la plus puissante des colonies italiotes du IV ${ }^{\mathrm{e}}$ siècle, Tarente, fait à des condottieri étrangers pour renforcer et guider ses armées dans les conflits qui l'opposent d'abord aux Lucaniens au IV ${ }^{\mathrm{e}}$ siècle, puis à Rome avec l'expédition de Pyrrhos au III siècle (Atti Taranto 2003 ; Atti Taranto 2004). La Grande-Grèce est en effet la première région occupée par des populations grecques à tomber sous la domination romaine, avec notamment les cas de Poseidonia, devenue colonie de droit latin en 273, et de Tarente, prise en 272 av. J.-C. Tout en gardant une certaine forme d'identité grecque, ces cités passent sous contrôle des Romains et rentrent dans l'optique des événements liés à l'histoire romaine dès le premier tiers du III siècle.

\section{CONCLUSION}

Grâce à un ensemble important de données archéologiques publiées, la région prise en considération, la Grande-Grèce, permet d'avoir un éclairage sur certains aspects essentiels de l'organisation des cités grecques. Pour l'époque archaïque, il s'agit certainement des cas d'études les plus développés sur une certaine préoccupation dans l'occupation des espaces, autant urbains que ruraux. Cet aspect spatial est indissociable de la question plus large de la formation de la cité grecque, la polis, aussi bien dans sa définition en tant qu'une communauté indépendante, que dans son expression sur le terrain, sous la forme d'un centre urbanisé entouré de terres cultivables. Les exemples occidentaux montrent que cette préoccupation dans la détermination des espaces, et de surcroît la volonté de régularisation, est presque contemporaine de l'installation coloniale. Du moins, les traces visibles et retrouvées par l'archéologie datent d'environ une génération après l'arrivée des nouveaux colons, aussi bien pour le centre urbain de Mégara Hyblaea, mais probablement aussi pour Cumes, que pour le territoire de Métaponte. C'est également dans le domaine occidental que l'on retrouve l'exemple certainement le plus abouti, attesté par les sources écrites et archéologiques, d'un urbanisme parfaitement orthogonal à Thourioi.

La préoccupation relative aux espaces, notamment sur les territoires, est indissociable de l'interaction avec les indigènes. Sybaris montre une cité grecque puissante qui se crée un réseau hégémonique (ou impérialiste) qui contrôle une large portion de l'Italie du Sud, surtout si l'on inclut les 
fondations secondaires de Métaponte et de Poseidonia. Ce contrôle n'est pas uniforme et fait état d'une graduation importante dans les rapports avec les éléments indigènes, de la conquête et soumission à des formes variées de cohabitation.

Enfin, pour une discussion sur les différentes formes de colonisation, il est essentiel de souligner que la Grande-Grèce constitue le plus ancien cas d'une "décolonisation ", c'est-à-dire de la conquête des cités coloniales grecques par des populations locales, comme à Naples, à Poseidonia et à Laos. Le renversement des rapports de force entre les éléments locaux et coloniaux montre que les interactions interethniques sont toujours très complexes. 


\section{REFERENCES BIBLIOGRAPHIQUES}

Megale Hellas: nome e immagine. Atti del Convegno di studi sulla Magna Grecia, XXI, 1981, Tarente: Istituto per la Storia e l'Archeologia della Magna Grecia

Poseidonia-Paestum. Atti del Convegno di Studi sulla Magna Grecia, XXVII, 1987, Tarente-Paestum: Istituto per la Storia e l'Archeologia della Magna Grecia, 1992.

Siritide e Metapontino. Storie de due territori coloniali. Cahiers du Centre Jean Bérard, XX, 1991, Policoro: Centre Jean Bérard, 1998.

Sibari e la Sibaritide. Atti del convegno di studi sulla Magna Grecia, XXXII, 1992, Tarente: Istituto per la storia e l'archeologia della Magna Grecia

Alessandro il Molosso e i 'Condotieri' in Magna Grecia. Atti del Convegno di studi sulla Magna Grecia, XLIV, 2003, Tarente: Istituto per la Storia e l'Archeologia della Magna Grecia, 2004.

Tramonto della Magna Grecia. Atti del Convegno di studi sulla Magna Grecia, XLIV, 2004: Istituto per la Storia e l'Archeologia della Magna Grecia, 2005.

Da Italia a Italia. Le radici di un'identità. Atti del Convegno di studi sulla Magna Grecia, LI, 2011, Tarente: Istituto per la storia e l'archeologia della Magna Grecia

Gli etruschi e la Campania settentrionale. Atti del XXVI Convegno di studi etruschi ed italici, Caserta, Santa Maria Capua Vetere, Capua, Teano, 11-15 novembre 2007, 2011, Pisa: F. Serra

ADAMESTEANU, Dinu. " Metaponto. Appunti fotointerpretativi », Atti della Accademia nazionale dei Lincei. Notizie degli scavi di antichità, XIX, suppl., 1965, p. 179-184.

ALLEGRO, Nunzio (éd.). Himera. V, L'abitato: isolato II. i blocchi 1-4 della zona 1, Palerme: Università di Palermo, Dipartimento di beni culturali, 2008.

AMERUOSO, Michele. Megále Hellás: genesi, storia ed estensione del nome, coll. Studi. pubbl. dall'Istituto italiano per la storia antica, 61, Rome: Istituto italiano per la storia antica, 1996.

ASHERI, David. "Osservazioni sulle origini dell'urbanistica Ippodamea », Rivista Storica Italiana, LXXXVII, I, 1975 , p. 5-16

ASHERI, David. « Colonizzazione e decolonizzazione », in Salvatore SETTIS (éd.). I Greci. Storia, cultura, arte, società. 1. Noi e i Greci, Turin: G. Einaudi, 1996, p. 73-115.

BIFFI, Nicola (éd.). L'Italia di Strabone. Testo, traduzione e commento dei libri V e VI della Geografia, Genova: DARFICLET, 1988.

BINTLIFF, John L. "Territorial behaviour and the natural history of the Greek polis », in Eckart OLSHAUSEN et Holger SONNABEND (éds.). Grenze und Grenzland. Stuttgarter Kolloquium zur Historischen Geographie des Altertums, coll. Geographica historica, 7, 4, Amsterdam: A. M. Hakkert, 1994, p. 207-249. 
BINTLIFF, John L. " Pattern and process in the city landscapes of Boetia from geometric to late roman times», in Michèle BRUNET (éd.). Territoires des cités grecques. Actes de la table ronde internationale, BCH Suppléments, 34, 1999, Athènes, Athènes: École Française d'Athènes, 1991, p. 15-33.

BROCATO, Paolo (éd.). Studi sulla necropoli di Macchiabate a Francavilla Marittima (Cs) e sui territori limitrofi. Ricerche, Supplementi, 5, Arcavacata di Rende: Università della Calabria, 2014.

BRUNET, Michèle. « Le ricerche sulle chorai della Grecia insulare: un bilancio critico ». Problemi della chora coloniale dall'Occidente al Mar Nero, Atti del Convegno di Studi sulla Magna Grecia, XL, 2000, Tarente, Naples: Istituto per la Storia e l'Archeologia della Magna Grecia, 2001, p. 27-45.

BUGNO, Maurizio. "Strabone VI, 1, 13 C. 263 e l'ápxń di Sibari », in Maurizio BUGNO et Concetta MASSERIA (éds.). II mondo enotrio tra VI e V secolo a.C., coll. Atti dei seminari napoletani, 1996-1998, Quaderni di ostraka, 1, Naples: Loffredo, 2001, p. 303-327.

CAHILL, Nicholas. Household and city organization at Olynthus, New Haven: Yale university, 2002.

CARTER, Joseph Coleman. The chora of Metaponto: the necropoleis, Austin, TX, 1998.

CARTER, Joseph Coleman. Discovering the Greek countryside at Metaponto, Ann Arbor: University of Michigan press, 2006.

CARTER, Joseph Coleman et PRIETO, Alberto (éds.). The chora of Metaponto. 3, Archaeological field survey Bradano to Basento, 3 vols., Austin, Tx: University of Texas Press, 2011.

CASTAGNOLI, Ferdinando. "Sull'urbanistica di Thurii », La parola del passato, 26, 1971, p. 301-307.

CASTAGNOLI, Ferdinando. "Ancora sull'urbanistica di Thurii », La parola del passato, 28, 1973, p. 220-222.

CERCHIAI, Luca. I Campani, Milan: Longanesi, 1995.

CERCHIAl, Luca. "La Campania: i fenomeni di colonizzazione ", in Giuseppe M. DELLA FINA (éd.). La colonizzazione etrusca in Italia. Atti del XV convegno internazionale di studi sulla storia $e$ I'archeologia dell'Etruria, coll. Annali della Fondazione per il Museo "Claudio Faina", XV, Roma: Quasar, 2008, p. 401-421.

CERCHIAl, Luca. Gli antichi popoli della Campania, coll. Studi superiori. archeologia, 598, Rome: Carocci editore, 2010.

CIPRIANI, Marina et LONGO, Fausto (éds.). I Greci in Occidente: Poseidonia e i Lucani, Naples, 1996.

DAVIES, John Kenyon. « La discussione sulla polis greca oggi », in Poleis e politeiai nella Magna Grecia arcaica e classica. Atti del Convegno di studi sulla Magna Grecia, Tarente: Istituto per la Storia e l'Archeologia della Magna Grecia, 2013, p. 11国33.

D'ACUNTO, Matteo. "L'abitato antico di Cuma tra le Terme del Foro e le mura settentrionali: relazione preliminare della campagna di scavo del 2007 dell'Università L'Orientale di Napoli », in Carlo GASPARRI et Giovanna GRECO (éds.). Cuma: indagini archeologiche e nuove scoperte, coll. Atti della 
giornata di studi, Napoli, 12 dicembre 2007. Quaderni del Centro studi Magna Grecia, 7. Studi cumani, 2, Pozzuoli: Naus, 2009, p. 73-87.

D'ACUNTO, Matteo. « Gli scavi dell'università degli studi di Napoli "L'Orientale" nell'abitato greco-romano di Cuma (2007-2013) », Newsletter di Archeologia CISA, 5, 2014, p. 21-38.

D'AGOSTINO, Bruno. " Gli Etruschi e gli altri nella Campania Settentrionale ». Gli Etruschi e la Campania settentrionale, coll. Atti del XXVI Convegno di studi etruschi ed italici, Caserta, Santa Maria Capua Vetere, Capua, Teano, 11-15 novembre 2007. Convegno di studi etruschi ed italici, 26, Pisa: F. Serra, 2011, p. 69-91.

D'AGOSTINO, Bruno et D'ACUNTO, Matteo. « La città e le mura: nuovi dati dall'area Nord della città antica ». Cuma. Atti del Convegno di Studi sulla Magna Grecia, XLVIIII, 2008, Tarente, 2009, p. 481-522.

D'ONOFRIO, Adele. « Primi dati sull'urbanistica di Cuma: I'area tra il foro e le fortificazioni settentrionali », in Bruno D'AGOSTINO et Andrea D'ANDREA (éds.). Cuma: nuove forme di intervento per lo studio del sito antico, coll. Atti della giornata di studio, Napoli 12 febbraio 2001, Naples: Istituto Universitario Orientale, 2002, p. 133-152.

DE ANGELIS, Franco. Megara Hyblaia and Selinous: the development of two Greek city-states in archaic Sicily, coll. OUSA Monographs, Oxford: Oxford University Press, 2003.

DE POLIGNAC, François. La Naissance de la cité grecque. Cultes, espace et société, VIII'-VII siècles, 2 e éd., Paris: La Découverte, 1995.

DE SIENA, Antonio. "Metaponto: problemi urbanistici e scoperte recenti ». Siritide e Metapontino. Storie di due territori coloniali, Cahiers du Centre Jean Bérard, XX, 1991, Policoro, Naples-Paestum: Centre Jean Bérard, 1998, p. 141-170.

DELIA, Giorgio et MASNERI, Tullio (éds.). Sibari: archeologia, storia, metafora. Quaderni del liceo, 2, Castrovillari: Il coscile, 2013.

DI VITA, Antonino. "L'Urbanisme de la Sicile grecque ", in Giovanni Pugliese CARRATELLI (éd.). Grecs en Occident. De l'âge mycénien à la fin de l'Hellénisme, coll. Catalogue de l'exposition du Palazzo Grassi de Venise. , Milan: Bompiani, 1996, p. 263-308.

ESPOSITO, Arianna. « Entre Sybaris et Tarente: archéologie d'une frontière. Identités, mythes et territoires dans le Golfe de Tarente (IXe-Ve s. av. J.-C.). », Université de Paris I, 2005.

GARCÍA QUINTELA, Marco. "Hipódamo en Turios: urbanismo, religión y política », Dialogues d'Histoire Ancienne, 26, 1, 2000, p. 7-33.

GRANESE, Maria Tommasa. "Culto e pratiche rituali nel santuario arcaico di Francavilla Marittima (Sibari CS) ", Annuario della Scuola archeologica di Atene e delle missioni italiane in oriente, LXXXIV, s. III, 6, t. 1, 2006, p. 417-463. 
GRANESE, Maria Tommasa. « Un luogo di culto del territorio di Sibari: il santuario di Francavilla Marittima (CS) », in Giorgio DELIA et Tullio MASNERI (éds.). Sibari: archeologia, storia, metafora, coll. Quaderni del liceo, 2, Castrovillari: II coscile, 2013, p. 57-84.

GRAS, Michel, TRÉZINY, Henri et BROISE, Henri. Mégara Hyblaea. 5, La ville archaïque: l'espace urbain d'une cité grecque de Sicile orientale, coll. Mélanges d'archéologie et d'histoire. Suppléments, 1, Rome: École française de Rome, 2004.

GRECO, Emanuele. "Strabone e la topografia storica della Magna Grecia », in Gianfranco MADDOLI (éd.). Strabone. Contributo allo studio della personalità e dell'opera, 2. Strabone e I'Italia antica, 1987, Acquasparta, Naples: Edizioni Scientifiche Italiane, 1988, p. 121-134.

GRECO, Emanuele. "L'impero di Sibari: bilancio archeologico-topografico ». Sibari e la Sibaritide. Atti del convegno di studi sulla Magna Grecia, XXXII, 1992, Tarente, Naples: Istituto per la Storia e l'Archeologia della Magna Grecia, 1993, p. 459-485.

GRECO, Emanuele. « Dalla Ionia alla Magna Grecia: Ippodamo di Mileto tra utopia e prassi ». Magna Grecia e Oriente mediterraneo prima dell'età ellenistica, Atti del convegno di studi sulla Magna Grecia, XXXIX, 1999, Tarente: Istituto per la storia e l'archeologia della Magna Grecia, p. 575-584.

GRECO, Emanuele. "Nomi di strade nelle città greche ", in MARINA CASTOLDI (éd.). Koina: miscellanea di studi archeologici in onore di Piero Orlandini, Milan: Edizioni ET, 1999, p. 223-229.

GRECO, Emanuele. "Turi », in Emanuele GRECO (éd.). La Città greca antica: istituzioni, società e forme urbane, Rome: Donzelli editore, 1999, p. 413-430.

GRECO, Emanuele. "Poseidonia-Paestum ", in André VAUCHEZ (éd.). Lieux sacrés, lieux de cultes, sanctuaires: approches terminologiques, méthodiques, historiques et monographiques, coll. Collection de l'École Française de Rome, 273, Rome: École Française de Rome, 2000, p. 81-94.

GRECO, Emanuele. « Note di topografia e di urbanistica », Annali di archeologia e storia antica, n.s. 11-12, $\mathrm{n}^{\circ}$ rassegne e recensioni, 2004-2005, p. 353-358.

GRECO, Emanuele. « Urban plan of Thourioi: literary sources and archaeological evidence for a Hippodamian city ", in Sara OWEN et Laura PRESTON (éds.). Inside the city in the Greek world: studies of urbanism from the Bronze Age to the Hellenistic period, Oxford: Oxbow books, 2009, p. 108-117.

GRECO, Emanuele. « Sul cosiddetto 'impero' di Sibari fino alla tirannide di Telys ed alla distruzione della città ", in Giorgio DELIA et TULLIO MASNERI (éds.). Sibari: archeologia, storia, metafora, coll. Quaderni del liceo, 2, Castrovillari: Il coscile, 2013, p. 197-203.

GRECO, Emanuele, GRECO, giovanna et PONTRANDOLFO, Angela. Da Poseidonia a Paestum, coll. Paestum. La città e il museo, 2, Salerne: Ingegneria per la cultura, s.d.

GRECO, Emanuele et LUPPINO, Silvana. « Ricerche sulla topografia e sull'urbanistica di Sibari-Thuri-Copiae », Annali di archeologia e storia antica, ns. 6, 1999, p. 115-164. 
GRECO, Emanuele; LUPPINO, Silvana, et alii. « Alla ricerca di Ippodamo di Mileto. L'impianto urbanistico di Thurii. La campagna di scavo 2003 a Sibari in località Lattughelle », Polis. Studi interdisciplinari sul mondo antico, 3, 2010, p. 97-116.

GRECO, Emanuele et TORELLI, Mario. Storia dell'urbanistica: il mondo greco, coll. Grandi Opere, Roma: Laterza, 1983.

GUGGISBERG, Martin A. « Local identity and cultural exchange in (pre-) colonial Francavilla Marittima: the Macchiabate necropolis in the light of new excavations », in Lieve DONNELLAN, Valentino NIZZO et Gert-Jan BURGERS (éds.). Contexts of early colonization, coll. Acts of the conference "Contextualizing early colonization: archaeology, sources, chronology and interpretative models between Italy and the Mediterranean", vol. 1. Papers of the Royal Netherlands Institute in Rome, 64, Roma: Palombi, 2016, p. 237-246.

GUZZO, Pier Giovanni. « Da Francavilla Marittima a Pithecusa », in Giuseppe ANDREASSI, assunta COCCHIARO et Antonietta DELL'AGLIO (éds.). Vetustis novitatem dare: temi di antichità e archeologia in ricordo di Grazia Angela Maruggi, Tarente: Scorpione, 2013, p. 81-88.

HANSEN, Morgens Herman. Polis et cité-État. Un concept antique et son équivalent moderne, Trad.Alexandre HASNOAOUI, Paris: Les Belles Lettres, 2001.

HANSEN, Morgens Herman et NIELSEN, Thomas Heine (éds.). An inventory of archaic and classical poleis: an investigation conducted by The Copenhagen Polis Centre for the Danish national research Foundation, Oxford: Oxford University Press, 2004.

HELLMANN, Marie-Christine L'architecture grecque. 3, Habitat, urbanisme et fortifications, coll. Les Manuels d'art et d'archéologie antiques, Paris: Picard, 2010.

JANNI, Pietro. "L'Italia di Strabone: descrizione e immagine », in Gianfranco MADDOLI (éd.). Strabone e I'Italia antica, Incontri perugini di storia della storiografia antica e sul mondo antico, 2, 1988, Acquasparta, Naples: Edizioni Scientifiche Italiane, p. 145-159.

LA GENIÈRE DE, Juliette. "Amendolara ", in Giuseppe NENCl et Georges VALLET (éds.). Bibliografia topografica della colonizzazione greca in Italia e nelle isole tirreniche, III, siti Abaceno-Bari, Pisa: Scuola normale superiore, 1984, p. 210-214.

LA GENIÈRE DE, Juliette. Amendolara: la nécropole de Paladino Ouest, coll. Collection du Centre Jean Bérard, 39, Naples: Centre Jean Bérard, 2012.

LA TORRE, Gioacchino Francesco. Sicilia e Magna Grecia. Archeologia della colonizzazione greca d'Occidente, coll. Manuali Laterza, Rome: Laterza, 2011.

LAFON, Xavier, MARC, Jean-Yves et SARTRE, Maurice. La Ville antique, coll. Histoire de l'Europe urbaine, 1. De I'Antiquité au XVIII' siècle, Paris: Seuil, 2011. 
LOMBARDO, Mario. « Achei, Enotri, Italia », in Emanuele GRECO (éd.). Gli Achei e l'identità etnica degli Achei d'Occidente, Atti del Convegno Internazionale di Studi, 2001, Paestum, Paestum: Pandemos, 2002, p. $257-270$.

LOMBARDO, Mario. « La Norma e l'eccesso: la guerra tra Sibari e Crotone e alcuni aspetti della 'Greek way of war' in età arcaica ", in Marta SORDI (éd.). Guerra e diritto nel mondo greco e romano, coll. Contributi dell'Istituto di storia antica, 28, Milan: Vita e Pensiero, 2002, p. 43-67.

LONGO, Fausto et TAURO, Teresa. Alle origini dell'urbanistica di Napoli, Paestum: Pandemos, 2017.

LUPPINO, Silvana; QUONDAM, Francesco, et alii. « Sibaritide: riletture di alcuni contesti funerari tra VIII e VII sec. a.C. ". Alle origini della Magna Grecia: mobilità, migrazioni, fondazioni. Atti del Convegno di studi sulla Magna Grecia, L, 2010, Tarente, Naples: Istituto per la Storia e I'Archeologia della Magna Grecia, p. 643-682.

MAASKANT-KLEIBRINK, Madeleine. « The early Athenaion at Lagaria (Francavilla Marittima) near Sybaris: an overview of its early-geometric II and its mid-7th century BC phases ». Papers in Italian archaeology. 6. Communities and settlements from the Neolithic to the Early Medieval Period. Proceedings of the 6th conference of Italian archaeology held at the University of Groningen, Groningen Institute of Archaeology, the Netherlands, April 15-17, 2003, BAR. International series, 1452, 2005, Oxford: Archaeopress, p. 754-772.

MADDOLI, Gianfranco. «Strabone e I'Italia antica. Dalla genisi della Geografia alla problematica dei libri V e VI », in Gianfranco MADDOLI (éd.). Strabone e I'Italia antica, 1987, Acquasparta, Naples: Edizioni Scientifiche Italiane, 1988, p. 9-22.

MARINO, Simone. Copia/Thurii: aspetti topografici e urbanistici di una città romana della Magna Grecia, coll. Tekmeria, 14, Paestum: Pandemos, 2010.

MARTIN, Roland. L'urbanisme dans la Grèce antique, coll. Grands manuels Picard, 2 e éd., Paris: Picard, 1974. MERTENS, Dieter. " L'Architettura e I'urbanistica di Metaponto nel quadro dell'economia locale e dell'evoluzione generale nella Magna Grecia ». Siritide e Metapontino. Storie di due territori coloniali, Cahiers du Centre Jean Bérard, XX, 1991, Policoro, Naples-Paestum: Centre Jean Bérard, 1998, p. 123-140.

MERTENS, Dieter. Città e monumenti dei Greci d'Occidente: dalla colonizzazione alla crisi di fine V secolo a.C., Trad.Massimiliano PAPINI, Rome: L'Erma di Bretschneider, 2006.

MUSTI, Domenico. " L'idea di Megale Hellás ». Strabone e la Magna Grecia: città e popoli dell'Italia antica, Padoue: Ed. Programma, 1988, p. 61-94.

OSANNA, Massimo. Chorai coloniali da Taranto a Locri: documentazione archeologica e ricostruzione storica, Rome: Istituto Poligrafico e Zecca dello Stato, 1992. 
PERONI, Renato et TRUCCO, Flavia (éds.). Enotri e Micenei nella Sibaritide. Magna Graecia, 8, Tarente: Istituto per la storia e l'archeologia della Magna Grecia, 1994.

POLLINI, Airton. "Bibliographical note on the study of the territory in Magna Graecia ", Workshop di Archeologia Classica. Paesaggi, costruzioni, reperti, n³, 2006, p. 37-56.

POLLINI, Airton. " Limites et occupation de l'espace dans les colonies grecques du Sud de l'Italie », in Laurianne Martinez-SÈVE (éd.). Les diasporas grecques du VIII à la fin du III siècle av. J.-C., coll. Pallas, 89, Toulouse: Presses universitaires du Mirail, 2012, p. 123-142.

POLLINI, Airton. «Introduction: les problèmes de la citoyenneté et de l'exclusion chez les anciens Grecs », in Céline BORELLO et Airton POLLINI (éds.). Questions d'appartenance: les identités de l'Antiquité à nos jours, Paris: Éditions Orizons, 2015, p. 21-34.

POLLINI, Airton. "Représentations de la ville grecque idéale: expériences concrètes et réélaborations ", in Myriam CHOPIN et Francesco D'ANTONIO (éds.). Théâtralisation de l'espace urbain, Paris: Orizons, 2017, p. 97-120.

POLLINI, Airton. (à paraître) « La contribution de l'archéologie à la question des statuts: espaces funéraires, domestiques et politiques ", in Claudia MOATTI et Christel MÜLLER (éds.). Statuts personnels et espaces sociaux: questions grecques et romaines, Nanterre: Maison Archéologie \& Ethnologie RenéGinouvès

PONTRANDOLFO, Angela. I Lucani: etnografia e archeologia di una regione antica, Milan: Longanesi, 1982.

PONTRANDOLFO, Angela et D'AGOSTINO, Bruno. « Greci, Etruschi e Italici nella Campania e nella Lucania tirrenica ", in Françoise-Hélène MASSA-PAIRAULT (éd.). Crise et transformation des sociétés archaïques de I'Italie antique au V siècle av. J.-C., Collection de l'École Française de Rome, 137, 1987, Rome: École Française de Rome, 1990, p. 101-116.

PRONTERA, Francesco. « L'Italia meridionale di Strabone. Appunti tra geografia e storia », in Gianfranco MADDOLI (éd.). Strabone e I'Italia antica, Incontri perugini di storia della storiografia antica e sul mondo antico, 1987, Acquasparta, Naples: Edizioni Scientifiche Italiane, 1988, p. 93-109.

QUONDAM, Francesco. "La necropoli di Francavilla Marittima: tra mondo indigeno e colonizzazione greca », in Marco BETTELLI, Cecilia DE FAVERI et Massimo OSANNA (éds.). Prima delle colonie: organizzazione territoriale e produzioni ceramiche specializzate in Basilicata e in Calabria settentrionale ionica nella prima età del ferro, coll. Atti delle giornate di studio, Matera, 20-21 novembre 2007, Venosa: Osanna, 2009, p. 139-178.

QUONDAM, Francesco. «La Sibaritide prima e dopo la fondazione di Sibari », in Lieve DONNELLAN, Valentino NIZZO et Gert-Jan BURGERS (éds.). Contexts of early colonization, coll. Acts of the conference "Contextualizing early colonization: archaeology, sources, chronology and interpretative models 
between Italy and the Mediterranean", vol. 1. Papers of the Royal Netherlands Institute in Rome, 64, Roma: Palombi, 2016, p. 247-257.

ROUVERET, Agnès. « De la cité grecque à la ville lucanienne: images féminines et signes d'identité "citadine" à Poseidonia-Paestum », in B ANDENMATTEN, P BADINOU, et alii (éds.). Lieux de mémoire antiques et médiévaux. Texte, image, histoire: la question des sources, a contrario, 2012, p. 113-139.

SCHMIEDT, Giulio et CHEVALLIER, Raymond. "Caulonia e Metaponto. Applicazione della fotografia aerea in ricerche di topografia antica nella Magna Grecia », L'Universo, 2-5, 1959

SCHMIEDT, Giulio et CHEVALLIER, Raymond. "Photographie aérienne et urbanisme antique en GrandeGrèce: Caulonia, Métaponte ", Revue Archéologique, 1, 1960, p. 1-31.

SHIPLEY, Graham. "Little boxes on the hillside: Greek town planning, Hippodamos, and polis ideology », in Morgens Herman HANSEN (éd.). The imaginary polis, coll. Acts of the Copenhagen Polis Centre, 7, Copenhagen: Copenhagen polis center, 2005, p. 335-403.

STAZIO, Attilio. «Osservazioni sulla monetazione di Metaponto ». Metaponto, Atti del Convegno di studi sulla Magna Grecia, XIII, 1973, Tarente, Naples: Istituto per la Storia e l'Archeologia della Magna Grecia, 1975, p. 67-106.

TRÉZINY, Henri. "Lots et îlots à Mégara Hyblaea. Questions de métrologie ». La Colonisation grecque en Méditérannée occidentale. Actes de la rencontre scientifique en hommage à Georges Vallet, coll. Collection de l'École Française de Rome, 251, Rome: École Française de Rome, 1999, p. 141-183.

TRÉZINY, Henri. " Urbanisme et voirie dans les colonies grecques archaïques de Sicile orientale », Pallas. Revue d'études antiques, 58, 2002, p. 267-282

VALLET, Georges. «Topographie historique de Mégara Hyblaea et problèmes d'urbanisme colonial », MEFRA, $85,2,1983$, p. 641-647.

VASSALLO, Stefano. Himera, città greca: guida alla storia e ai monumenti, Palerme: Regione siciliana, 2005.

WEBER, Berthold F. " Der Stadtplan von Milet. Einhundert Jahre Stadtforschung ». Frühes Ionien: eine Bestandsaufnahme, Milesische Forschungen, 5, 2007, Mainz am Rhein: Ph. von Zabern, 1999, p. 327-362. 


\section{FIGURES}

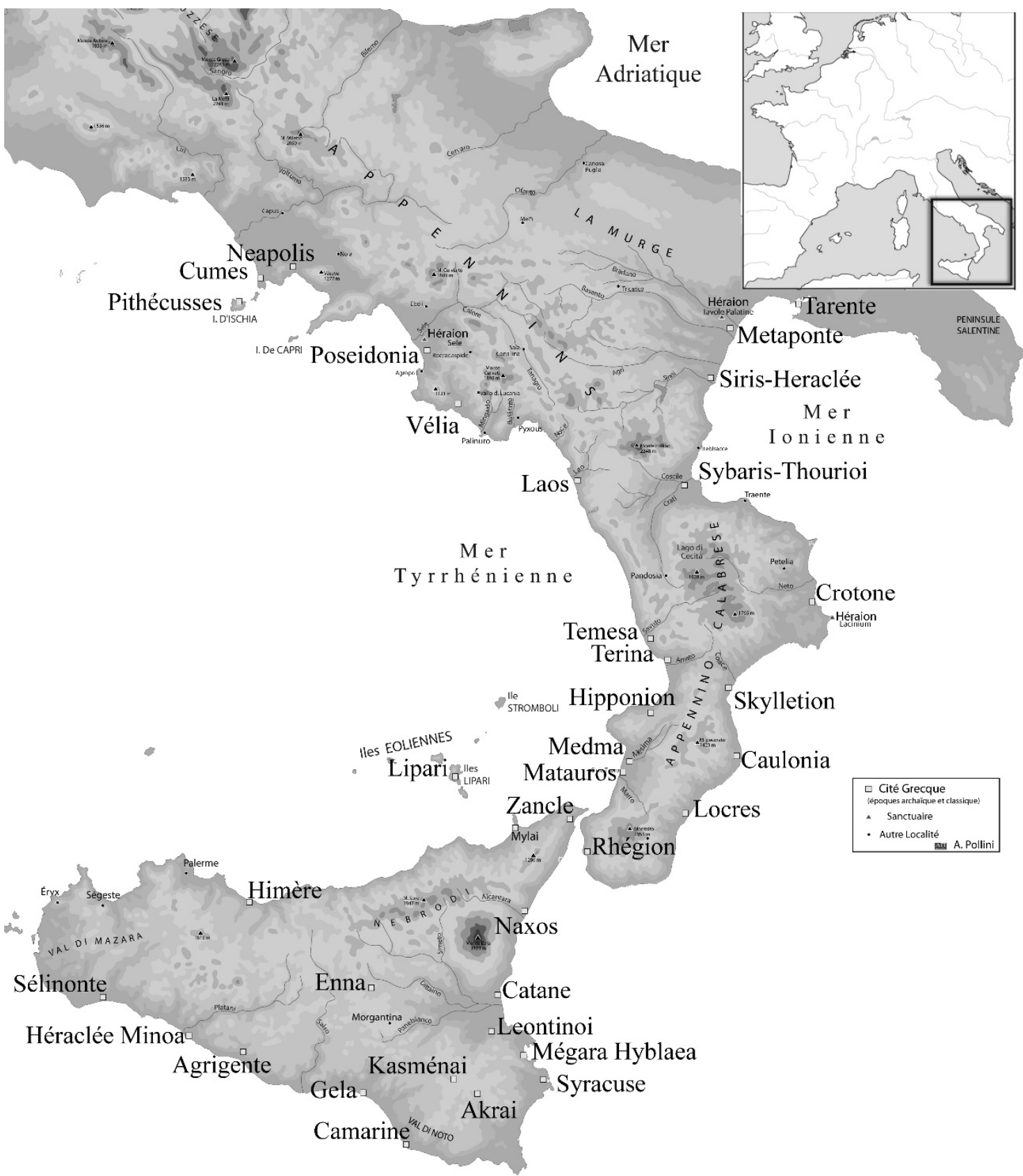

Fig. 01 : Carte de la Grande-Grèce et de la Sicile. (C) Airton Pollini 


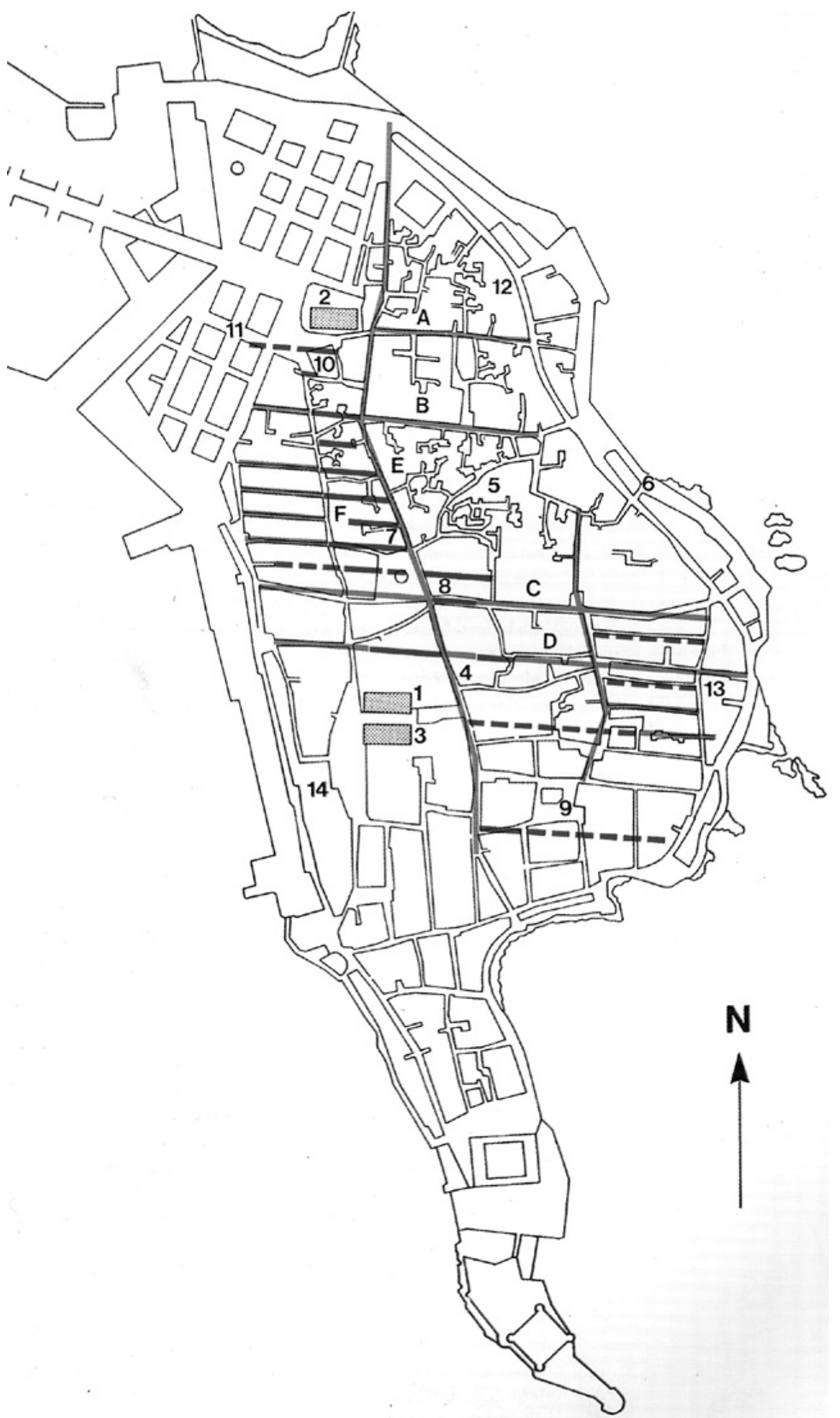

Fig. 02 : Plan urbain de l'île d'Ortygie, Syracuse. D’après Grecs en Occident : de l'âge mycénien à la fin de I'hellénisme, sous la direction de G. Pugliese Carratelli, publié à l'occasion de l'exposition "I Greci in Occidente" à Palazzo Grassi, Venise, Bompiani, 1996, p. 271. 


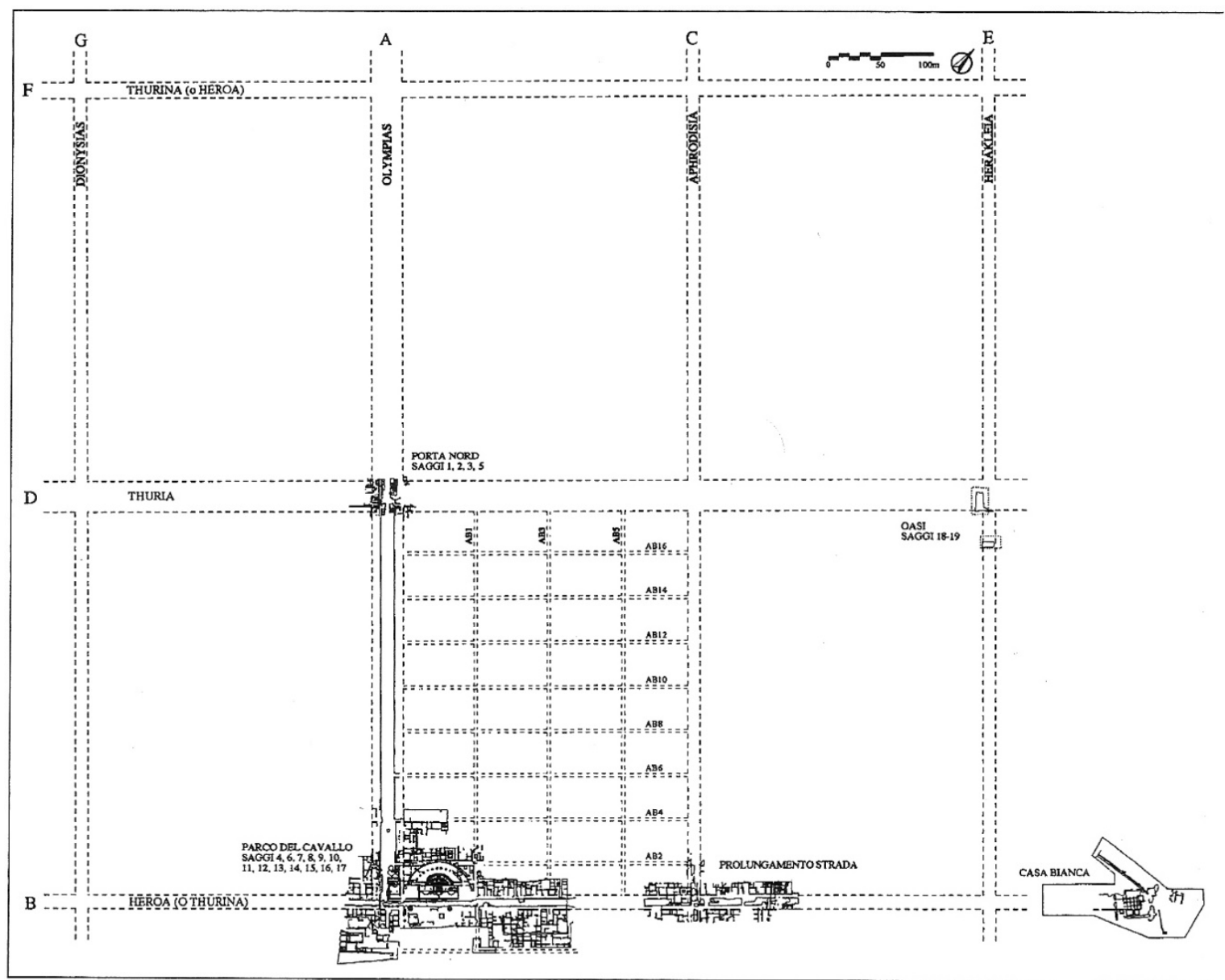

Figure 9.4 Reconstruction of the urban layout of Thourioi.

Fig. 03 : Reconstruction du plan urbain de Thourioi. D'après E. Greco, « Urban plan of Thourioi: literary sources and archaeological evidence for a Hippodamian city ", in S. Owen et L. Preston (org.). Inside the city in the Greek world: studies of urbanism from the Bronze Age to the Hellenistic period, Oxford: Oxbow books, 2009, fig. 9.4. 


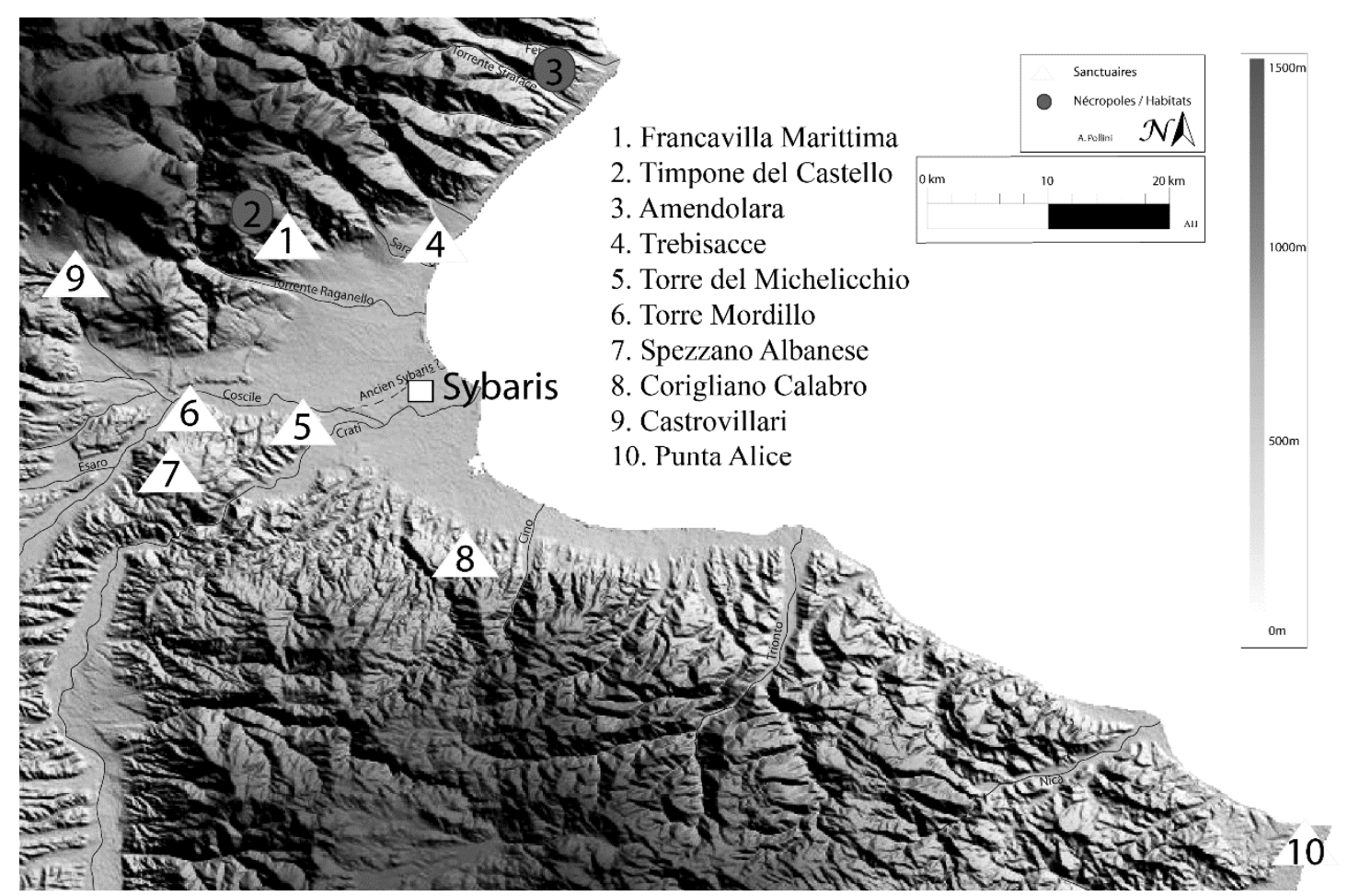

Fig. 04 : Carte du territoire de Sybaris, avec l'indication des principaux sites extra urbains de la colonie. (C) Airton Pollini

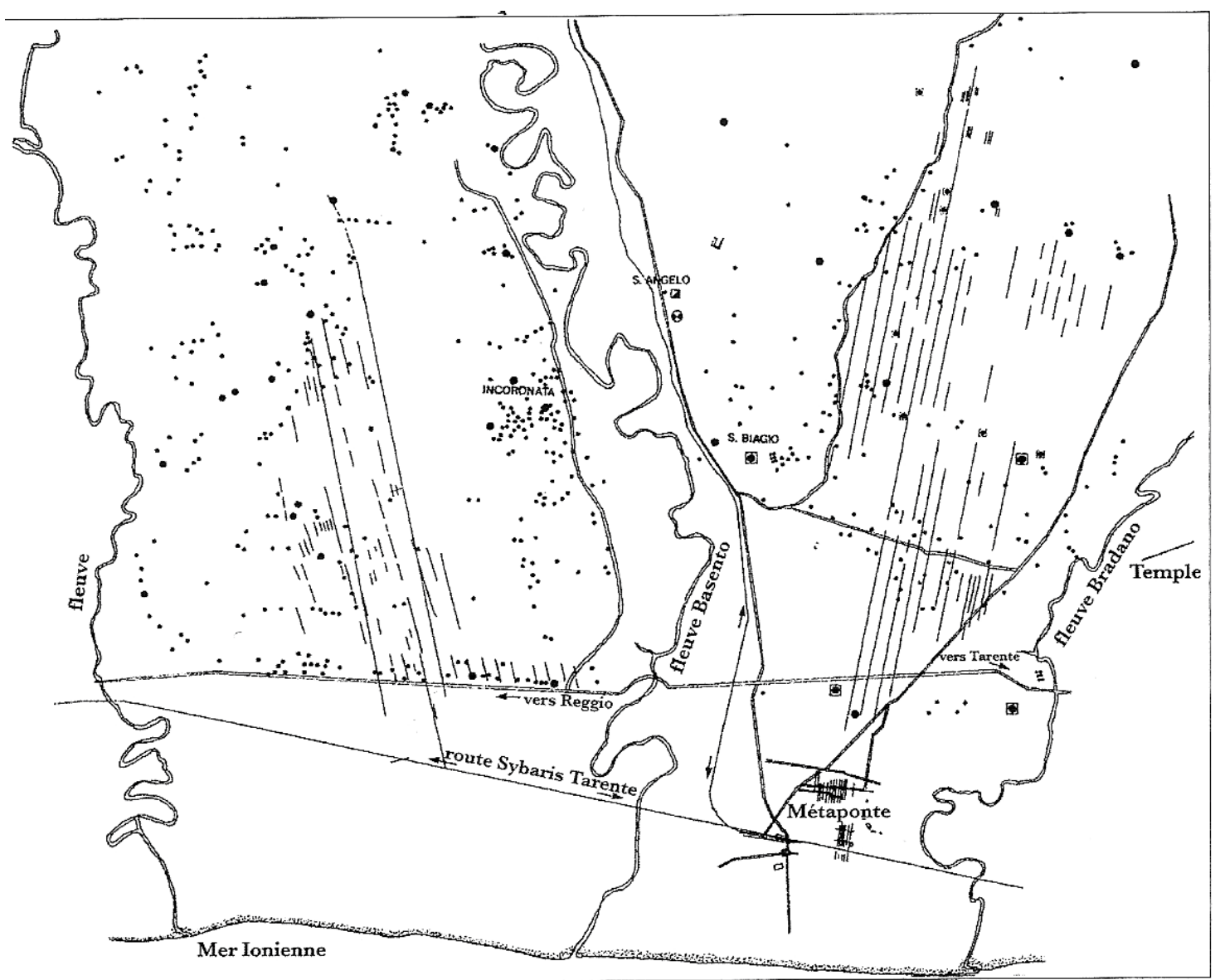

Fig. 05 : Carte avec division régulière de l'espace rural de Métaponte. D'après M.-Chr. Hellmann, L'architecture grecque. Vol. 3: Habitat, urbanisme et fortifications, Paris, Picard, 2010, fig. 286. 


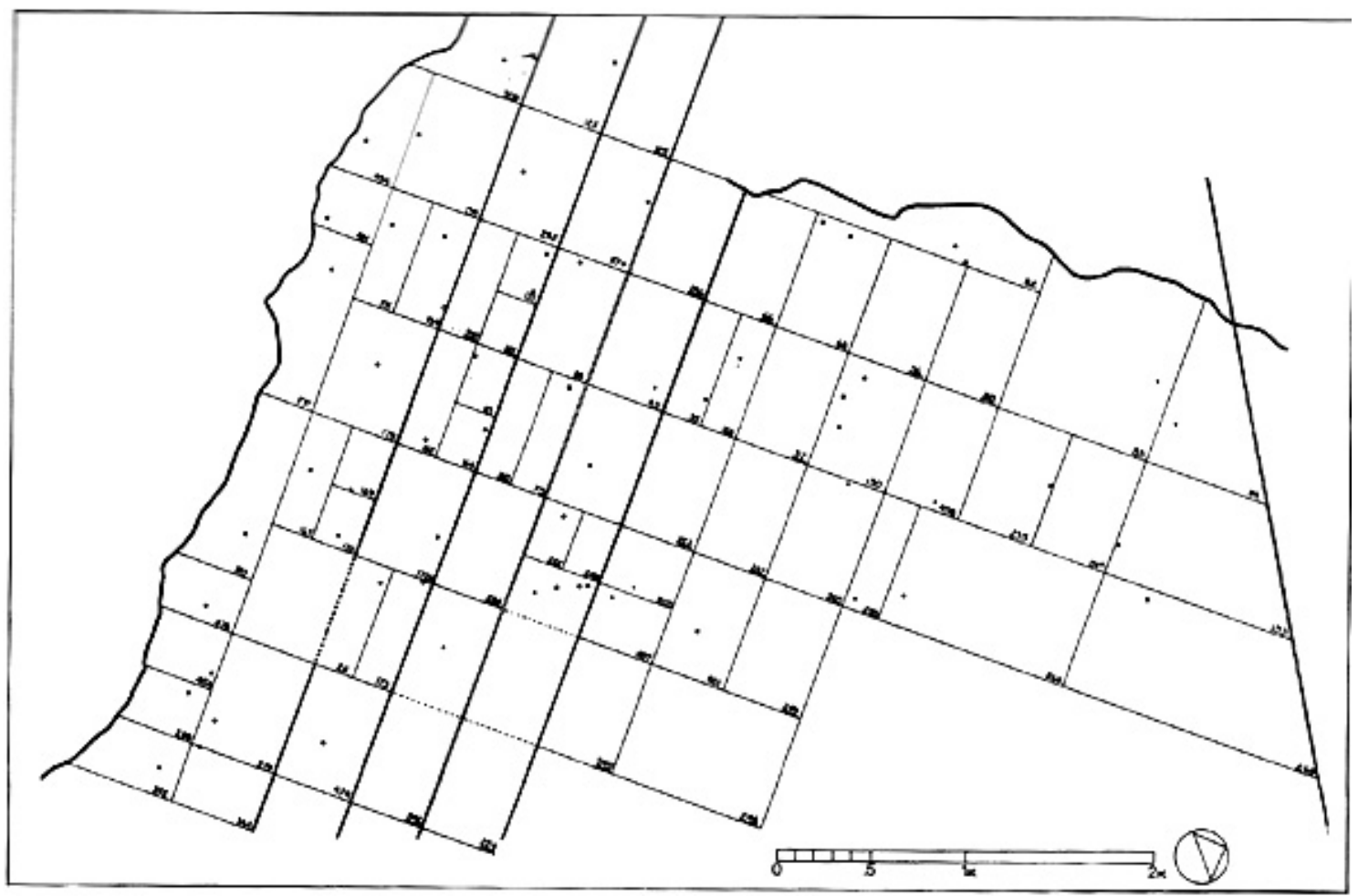

Fig. 06 : Lots de terre de Métaponte. D’après Grecs en Occident : de l'âge mycénien à la fin de l'hellénisme, sous la direction de G. Pugliese Carratelli, publié à l'occasion de l'exposition "I Greci in Occidente" à Palazzo Grassi, Venise, Bompiani, 1996, p. 247.
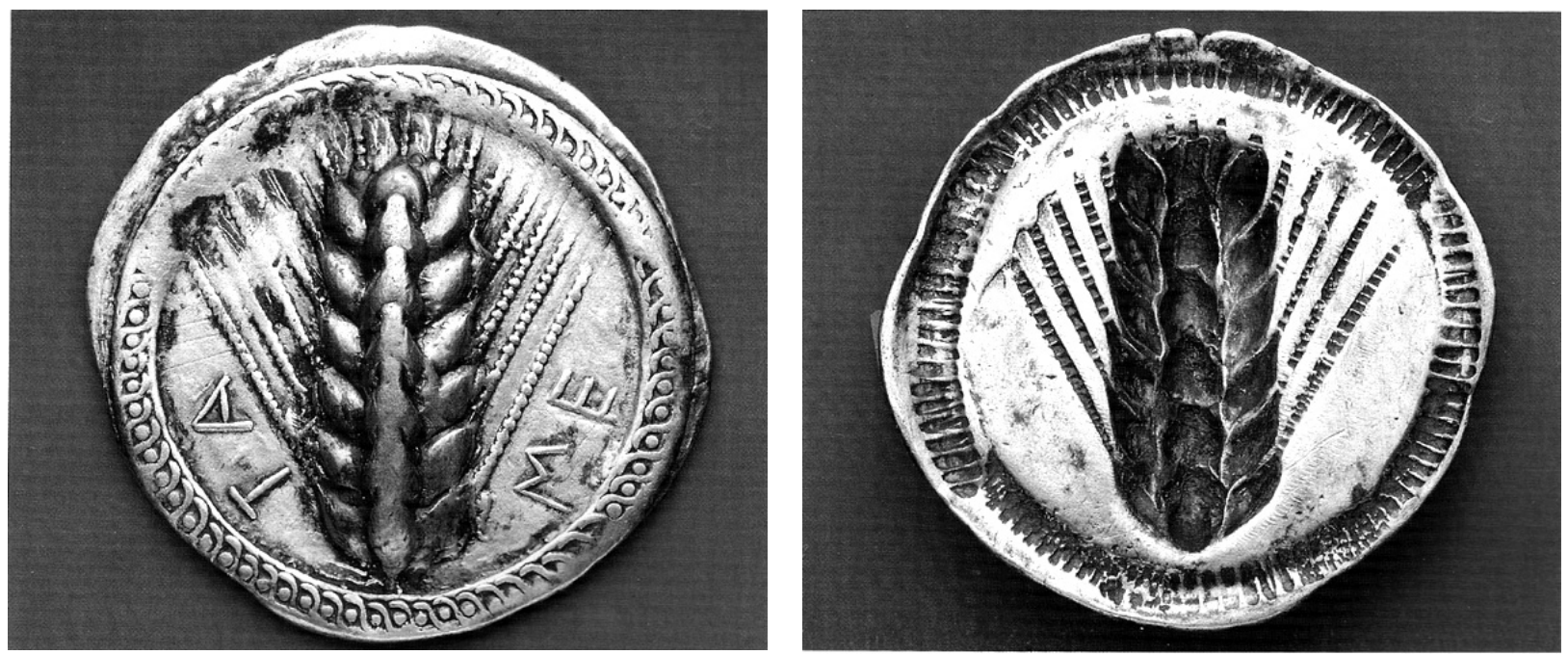

Fig. 07 : Statère d'argent de Métaponte représentant un épi d'orge. D'après Grecs en Occident : de l'âge mycénien à la fin de l'hellénisme, sous la direction de G. Pugliese Carratelli, publié à l'occasion de l'exposition "I Greci in Occidente" à Palazzo Grassi, Venise, Bompiani, 1996, p. 225.

Recebido em: 23/07/2017

Submited in: $23 / 07 / 2017$

Aprovado em: 04/09/2017

Aproved in: 04/09/2017

Publicado em: 24/06/2018

Published in: 24/06/2018 